\title{
Phosphonated Poly(ethylene terephthalate) lonomers as Compatibilizers in Polyester/Polyamide Blends for Packaging Applications
}

\author{
Lin Ju, Jeremy A. Beach, Ryan J. Mondschein, Juan Pretelt, Timothy E. Long, Donald G. Baird, and Robert B. \\ Moore* \\ Macromolecules Innovation Institute \\ Department of Chemistry \\ Virginia Tech \\ Blacksburg, VA 24060 \\ Phone: (540) 231-6875 \\ Email: linj7@vt.edu
}

\begin{abstract}
Poly(ethylene terephthalate) (PET) is widely used in the packaging industry. The oxygen barrier properties of PET are acceptable for many food and beverage products, but do not meet the stringent requirements for packaging highly oxygen-sensitive products. Blending PET with aromatic polyamides, such as poly ( $m$-xylyene adipamide) (MXD6), reduces the inherent oxygen permeability of the polyester matrix. Due to the immiscibility of these two parent polymers, a compatibilizer is necessary to achieve an efficient and stable mixing.

Herein, we present phosphonated PET (PPET) ionomer as a minor-component compatibilizer for PET/MXD6 melt blends [1-5]. Small amounts of ionomer enable the compatibilization of polymer blends through creating specific interactions between the ionic functionality and the amide units of MXD6, which lowers the interfacial tension and leads to a reduction in phase dimensions. PPET ionomer is synthesized by melt polycondensation, and the blends with various PPET ionomer loadings are extruded via a Leitritz twin screw extruder ( $L / D=40, D=18$ $\mathrm{mm}$ ). Blends properties including morphological, thermal, mechanical, and barrier properties are investigated in order to evaluate the compatibility of PPET ionomer. Using scanning electron microscope (SEM), we demonstrate decreased phase size of the dispersed MXD6 component in PET matrix as a result of added PPET ionomer. Haze value measurements indicate decreased haze value with increasing ionic content, which further confirms reduced MXD6 domain dimension. Thermal analysis shows increased nucleation effect of MXD6 on PET crystallization and inhibited crystallization of MXD6 in compatibilized blends. Compatibilized blends exhibit comparable mechanical properties to uncompatibilized blend. Oxygen permeability is studied to examine the gas barrier properties for both unoriented and oriented blends. Unoriented blends exhibit similar oxygen permeability, whereas a significant decrease is observed on oxygen permeability of the oriented films. Biaxial orientation transforms spherical MXD6 domains into platelets oriented in the plane of the film, and an enhanced barrier is achieved from the increased tortuosity of the diffusion pathway. The successful compatibilization of PET/MXD6 blends by utilizing PPET ionomer as compatibilizer offers potential for the next generation of blown bottles with high oxygen barrier property in packaging applications.
\end{abstract}

(a) PET/MXD6

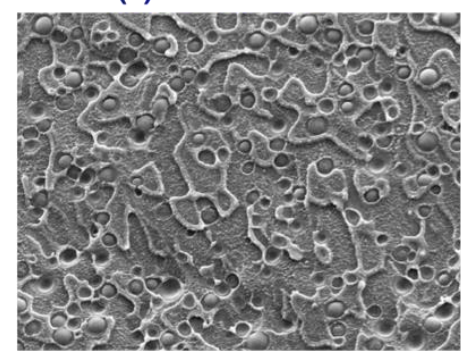

Scanning Electron Microscope of (a) uncompatibilized blend, and (b) compatibilized blend.

(b) PET/PPET/MXD6

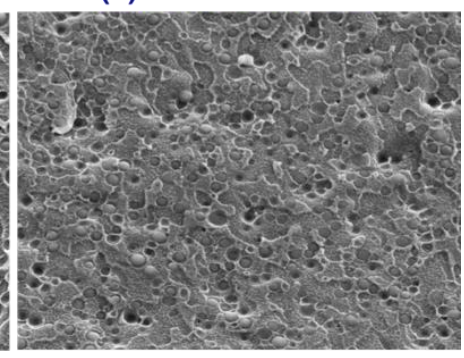

要 


\section{Reference}

1. Ju, L.; Pretelt, J.; Chen, T.; Dennis, J. M.; Heifferon, K. V.; Baird, D. G.; Long, T. E.; Moore, R. B. J. P., Synthesis and characterization of phosphonated poly (ethylene terephthalate) ionomers. 2018, 151, 154-163.

2. Ju, L.; Mondschein, R. J.; Vandenbrande, J. A.; Arrington, C. B.; Long, T. E.; Moore, R. B. J. P., Phosphonated poly (ethylene terephthalate) ionomers as compatibilizers in extruded poly (ethylene terephthalate)/poly (m-xylylene adipamide) blends and oriented films. 2020, 205, 122891.

3. Ju, L. Non-covalent interactions in polymeric materials: From ionomers to polymer blends. Virginia Tech, 2019.

4. Ju, L.; Dennis, J.; Valentine, K.; Long, T.; Moore, R. In Compatibilization of polyester/polyamide blends: Phosphonated polyester ionomer as minor-component compatibilizer with divalent anion, ABSTRACTS OF PAPERS OF THE AMERICAN CHEMICAL SOCIETY, AMER CHEMICAL SOC 1155 16TH ST, NW, WASHINGTON, DC 20036 USA: 2018.

5. Ju, L.; Dennis, J. M.; Heifferon, K. V.; Long, T. E.; Moore, R. B. J. A. A. P. M., Compatibilization of polyester/polyamide blends with a phosphonated poly (ethylene terephthalate) ionomer: Comparison of monovalent and divalent pendant ions. 2019, 1, 10711080. 